\title{
The Xanthomonas campestris pv. vesicatoria Type III Effector Protein XopJ Inhibits Protein Secretion: Evidence for Interference with Cell Wall-Associated Defense Responses
}

\author{
Verena Bartetzko, ${ }^{1}$ Sophia Sonnewald, ${ }^{1}$ Florian Vogel, ${ }^{1}$ Kristina Hartner, ${ }^{1}$ Ruth Stadler, ${ }^{2}$ \\ Ulrich Z. Hammes, ${ }^{2}$ and Frederik Börnke ${ }^{1}$ \\ ${ }^{1}$ Institut für Biologie, Lehrstuhl für Biochemie, Friedrich Alexander Universität Erlangen-Nürnberg, Staudtstr. 5, 91058 Erlangen, \\ Germany; ${ }^{2}$ Institut für Biologie, Lehrstuhl für Molekulare Pflanzenphysiologie, Friedrich Alexander Universität Erlangen- \\ Nürnberg, Staudtstr. 5, 91058 Erlangen, Germany
}

Submitted 24 July 2008. Accepted 13 February 2009.

The phytopathogenic bacterium Xanthomonas campestris pv. vesicatoria uses the type III secretion system (T3SS) to inject effector proteins into cells of its Solanaceous host plants. It is generally assumed that these effectors manipulate host pathways to favor bacterial replication and survival. However, the molecular mechanisms by which type III effectors suppress host defense responses are far from being understood. Based on sequence similarity, Xanthomonas outer protein $\mathbf{J}$ (XopJ) is a member of the YopJ/ AvrRxv family of SUMO peptidases and acetyltranferases, although its biochemical activity has not yet been demonstrated. Confocal laser scanning microscopy revealed that green fluorescent protein (GFP) fusions of XopJ are targeted to the plasma membrane when expressed in plant cells, which most likely involves $\mathrm{N}$-myristoylation. In contrast to a $\operatorname{XopJ}(\mathbf{C 2 3 5} \mathrm{A})$ mutant disrupted in the catalytic triad sequence, the wild-type effector GFP fusion protein was also localized in vesicle-like structures colocalizing together with a Golgi marker protein, suggesting an effect of XopJ on vesicle trafficking. To explore an effect of XopJ on protein secretion, we used a GFP-based secretion assay. When a secreted (sec)GFP marker was coexpressed with XopJ in leaves of Nicotiana benthamiana, GFP fluorescence was retained in reticulate structures. In contrast, in plant cells expressing secGFP alone or along with the XopJ(C235A) mutant, no GFP fluorescence accumulated within the cells. Moreover, coexpressing secGFP together with XopJ led to a reduced accumulation of secGFP within the apoplastic fluid of $N$. benthamiana leaves, further showing that XopJ affects protein secretion. Transgenic expression of XopJ in Arabidopsis suppressed callose deposition elicited by a T3SS-negative mutant of Pseudomonas syringae pv. tomato DC3000. A role of XopJ in the inhibition of cell wall-based defense responses is discussed.

Current address for U. Z. Hammes and F. Börnke: Cell Biology and Plant Physiology, University of Regensburg, 93053 Regensburg, Germany

Corresponding author: F. Börnke;

E-mail: fboernke@biologie.uni-erlangen.de

*The $e$-Xtra logo stands for "electronic extra" and indicates a supplemental figure is published online.
Xanthomonas campestris pv. vesicatoria is the causal agent of bacterial spot disease on pepper and tomato plants. Like many other phytopathogenic bacteria, the ability of $X$. campestris pv. vesicatoria to cause disease relies on the type III secretion system (T3SS) encoded by a cluster of hrp genes (Büttner and Bonas 2006). Bacteria inject a suite of type III effector proteins through the T3SS directly into the cytosol of the host cell (Galán and Wolf-Watz 2006). Bacterial mutants lacking a functional T3SS are nonpathogenic, indicating that successful establishment of disease requires translocation of type III effectors (Alfano and Collmer 2004). The analysis of the complete genome sequence of $X$. campestris pv. vesicatoria 85-10 led to the identification of at least 20 type III effectors (Thieme et al. 2005) of which, up to now, 17 have been experimentally verified (Gürlebeck et al. 2006; Thieme et al. 2007). Cumulatively, these effectors alter host processes to optimize the inter- and intracellular environment for pathogen growth while they promote disease development through suppression of host defense responses. The precise mechanisms by which type III effectors achieve this are largely unknown. Functional analysis of type III effectors has been hampered due to the fact that deletion of individual proteins by mutagenesis rarely has a measurable effect on bacterial fitness such as bacterial multiplication, symptom development, or colony formation (Noël et al. 2003), suggesting redundancy of effector function in bacterial pathogenicity.

Although the primary function of type III effectors is to promote plant susceptibility, considerable knowledge concerning the action of these proteins in plant cells comes from the studies of effectors classified as avirulence (Avr) proteins (White et al. 2000). Upon translocation into the plant cell, these proteins may be recognized by the corresponding plant disease resistance $(\mathrm{R})$ proteins in resistant plants and trigger defense responses, including the hypersensitive response, a rapid, localized, programmed, cell-death reaction eventually restricting bacterial growth (Staskawicz 2001). The fact that Avr effectors are maintained in most bacterial populations suggests that the primary function of these proteins is in establishment of the infection, i.e., virulence (Grant et al. 2006; Mudgett 2005). For example, AvrBs3, initially isolated based on its avirulence activity on resistant pepper plants (Bonas et al. 1989), induces hypertrophy symptoms in susceptible plants, i.e., an enlargement of mesophyll cells (Marois et al. 2002). Intriguingly, AvrBs3 has characteristic features of eukaryotic transcription 
factors, suggesting that it modulates the host's transcriptome. Recently, it was shown that AvrBs3 directly binds to the promoter region of target genes and activates transcription of the pepper resistance gene $B S 3$ in resistant plants (Römer et al. 2007), while it induces the expression of a master regulator of cell size in susceptible plants (Kay et al. 2007). Thus, AvrBs3 provokes reprogramming of the host's transcriptome by mimicking eukaryotic transcription factors.

Several type III effectors have been shown to possess enzymatic activity (Grant et al. 2006), e.g., HopPtoD2 from Pseudomonas syringae is a tyrosine phosphatase (Espinosa et al. 2003), while AvrPtoB from the same bacterium has E3 ubiquitin ligase activity (Janjusevic et al. 2006). Another example from $P$. syringae is HopU1, which has been shown to possess ADP-ribosyl transferase activity (Fu et al. 2007). The respective enzymatic activities of these type III effectors are correlated with their ability to either trigger-specific $R$ gene-mediated defense reactions on resistant host plants or to quell defense responses on susceptible plants. Other $P$. syringae effectors such as HopM1, AvrPto, AvrRpm1, and AvrRpt2 have been shown to suppress defense readouts associated with basal defense (pathogen-associated molecular pattern [PAMP]-induced innate immunity) (de Torres et al. 2006; DebRoy et al. 2004; Hauck et al. 2003; Nomura et al. 2006; Oh and Collmer 2005; Xiang et al. 2008). Deposition of callose is one of the most striking responses during PAMP-triggered immunity. Callose is a structural component of papillae, which are defense-associated cell-wall thickenings that incorporate antimicrobial compounds (Bestwick et al. 1995). X. campestris pv. vesicatoria appears to also possess type III effector proteins capable of suppressing basal defense responses as the wild-type strain does not induce papilla formation, while the respective type III-deficient $h r p$ mutant fails to suppress cell wall-associated defense responses upon infection (Brown et al. 1995). However, which type III effectors might be involved in this process in the case of $X$. campestris pv. vesicatoria remains to be determined.

XopJ (Xanthomonas outer protein $\mathbf{J}$ ) is a member of a family of type III effectors called YopJ-like proteins that are found in a wide variety of pathogens including plant pathogens, animal pathogens, and the plant symbiont Rhizobium spp. (Grant et al. 2006; Orth 2002). In Xanthomonas campestris pv. vesicatoria strains, four YopJ-like proteins were identified, i.e., XopJ, AvrRxv, AvrXv4, and AvrBsT (Hotson and Mudgett 2004). The founding member of the family, YopJ from Yersinia spp., inhibits the mitogen-activated protein kinase (MAPK) and nuclear factor $-\kappa \mathrm{B}$ signaling cascades within mammalian host cells, thereby inhibiting the host's innate immune response. All YopJ-like proteins possess a catalytic triad and, based on homology of YopJ to Clan CE cysteine proteases, it was initially proposed that it functions as a de-SUMOylating enzyme, thereby disrupting host signal transduction (Orth 2002). The expression of YopJ in animal cells inhibits the conjugation of SUMO to target proteins (Orth et al. 2000). However, recent work showed that YopJ functions as an acetyltransferase acetylating the critical serine and threonine residues found on the activation loop of MAPK kinases, thereby blocking their activation by phosphorylation (Mukherjee et al. 2006, 2007). Whether other YopJ-like proteins generally possess acetyltransferase activity or SUMO protease activity or both is currently not known. Interestingly, AvrXv4 expression in planta led to a reduction in SUMO-modified proteins, demonstrating that AvrXv4 possess SUMO isopeptidase activity (Roden et al. 2004). For XopJ, it is neither known whether it has isopeptidase activity nor which cellular process it might target. A XopJ mutant strain was not affected in bacterial growth and symptom formation on susceptible pepper plants, indicating subtle contribution of XopJ to bacterial virulence or functional redun- dancy (Noël et al. 2003). In a recent study, Thieme and associates (2007) were able to demonstrate myristoylation site-dependent localization of a XopJ::GFP fusion protein to the plasma membrane of plant cells and the ability of transiently expressed XopJ to elicit cell death in Nicotiana benthamiana. To shed further light on possible XopJ functions, we used Agrobacterium-mediated expression of XopJ variants in leaves of $N$. benthamiana. Transient expression of XopJ variants fused to a GFP reporter confirmed localization of the protein to the plasma membrane but implied that the fusion protein also colocalized with a Golgi marker protein. Microscopic analysis of XopJ-expressing plant cells suggested that the effector affects vesicle trafficking. Using a secreted version of GFP (secGFP) as a reporter, we were able to show that XopJ interferes with protein secretion. This effect required a functional catalytic triad. A possible role of XopJ in the suppression of polarized basal defense is discussed.

\section{RESULTS}

\section{XopJ::GFP localizes to the plasma membrane and in Golgi vesicles when transiently expressed in epidermal cells of $N$. benthamiana.}

To characterize the role of XopJ in planta, GFP was fused to the protein's $C$ terminus in a binary vector under control of the Cauliflower mosaic virus $35 \mathrm{~S}$ promoter. Subsequently, the XopJ::GFP fusion protein was transiently expressed in leaves of $N$. benthamiana plants, using Agrobacterium-mediated gene transfer. Next, the tissue was analyzed using confocal laser scanning microscopy of epidermal cells on the abaxial leaf side. At $48 \mathrm{~h}$ after infiltration, the majority of the inspected cells showed GFP fluorescence in the periphery of the cell, indicating plasma membrane localization of the fusion protein (Fig. 1). To further investigate this localization pattern, we coexpressed XopJ::GFP with mCherry-PM-rk, which localizes to the plasma membrane based on a fusion with AtPIP2A (Nelson et al. 2007). The results confirmed colocalization of XopJ::GFP and mCherry-PM-rk fluorescence (Fig. 1). In approximately $38 \%$ of the XopJ::GFP expressing cells analyzed (13 of 34 cells counted in three images), the GFP signal could also be detected in punctuate structures of varying sizes that resembled Golgi stacks (Fig. 1). At higher magnification, streaming of these punctuate structures was observed, confirming viability of the cells (data not shown). To determine whether these fluorescent structures represent Golgi stacks, we coexpressed XopJ::GFP with mCherry-G-rk, which localizes to the Golgi (Nelson et al. 2007). Overlay of GFP and mCherry fluorescence clearly showed that the XopJ::GFP-decorated punctuate structures overlapped with Golgi stacks (Fig. 1).

In order to investigate whether the fluorescence pattern observed upon expression of the XopJ::GFP wild-type protein could be attributed to its biological activity in plant cells, we fused GFP to the $\operatorname{XopJ}(\mathrm{C} 235 \mathrm{~A})$ mutant disrupted in the catalytic triad and analyzed localization of the fusion protein as described above. Confocal microscopy revealed that the $\mathrm{XopJ}(\mathrm{C} 235 \mathrm{~A}):$ :GFP fusion protein exclusively localized to the periphery of the cell, and coexpression of XopJ(C235A)::GFP with mCherry-PM-rk showed a clear localization of the fusion protein to the plasma membrane (Fig. 1). Interestingly, $\mathrm{XopJ}(\mathrm{C} 235 \mathrm{~A}):$ :GFP fluorescence was never observed in punctuate structures, as previously shown for the wild-type protein, and the mutated protein did not colocalize with a mCherryGolgi (Fig. 1), indicating that Golgi localization of XopJ::GFP might be associated with its biological activity.

Recently, it was shown that XopJ is localized to the host cell plasma membrane, possibly involving N-myristoylation (Thieme et al. 2007). When the predicted N-myristoylation of 


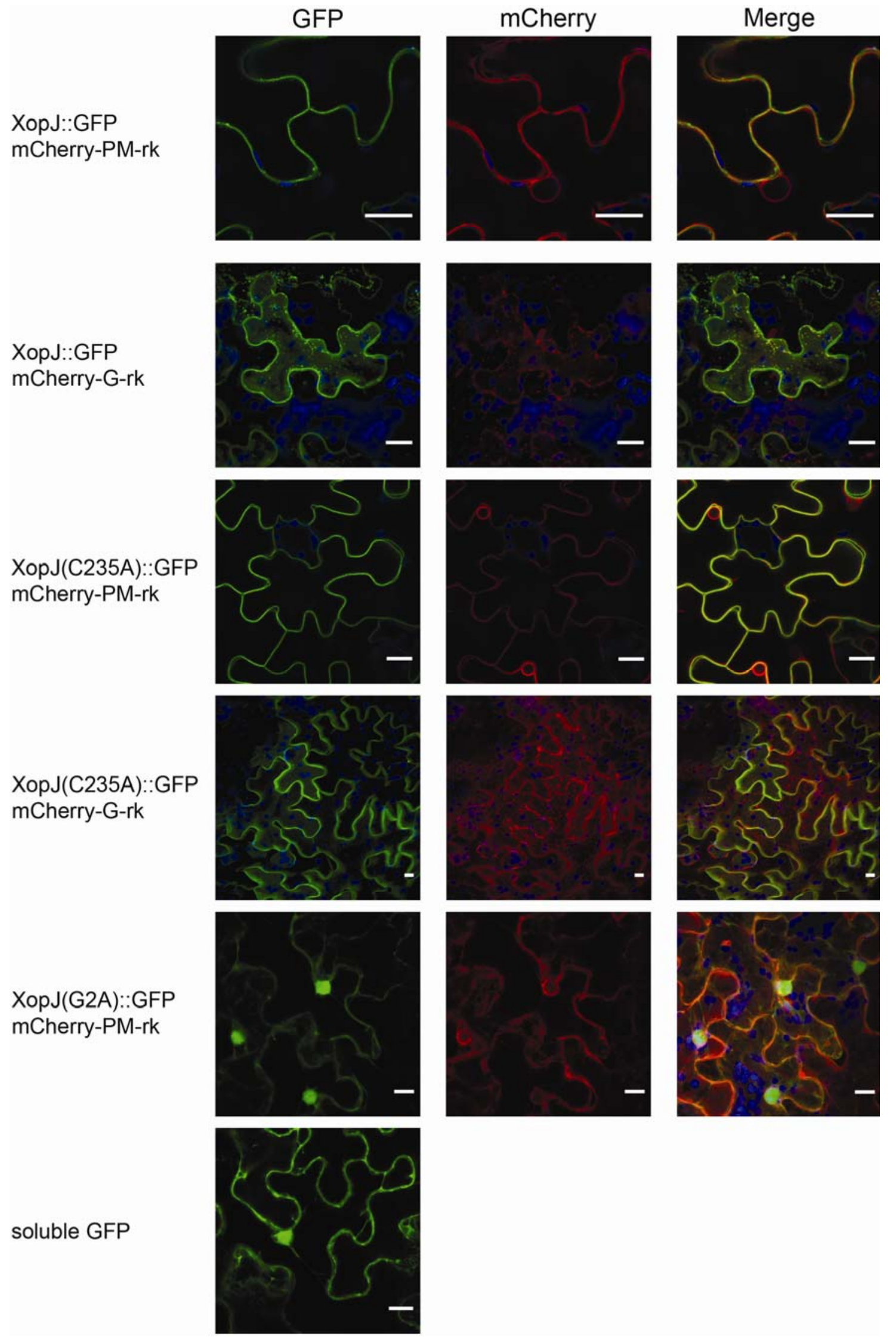

Fig. 1. The subcellular localization of XopJ::GFP (green fluorescent protein) depends on functional motifs. Agrobacterium strains that mediate T-DNAbased transfer of XopJ::GFP, XopJ(C235A)::GFP, and XopJ(G2A)::GFP, respectively, were infiltrated into Nicotiana benthamiana leaves together with mCherry markers for the plasma membrane (mCherry-PM-rk) and the Golgi (mCherry-G-rk) as indicated. For confocal laser scanning microscopy, samples were taken $48 \mathrm{~h}$ postinoculation, and fluorescent channels were scanned sequentially. GFP fluorescence is shown in green (left panel) and mCherry fluorescence in red (middle panel). The far right panels show a merged view of both fluorescence channels, including chlorophyll autoflourescence (blue). For comparison, the fluorescence pattern of free GFP is shown (left panel, bottom). The scale bar represents $15 \mu \mathrm{m}$. 
XopJ was rendered dysfunctional by exchanging the glycine residue at position two of the polypeptide to alanine, the XopJ (G2A)::GFP fusion protein appeared to be localized to the cytoplasm, cytoplasmic strands, and nuclei of infected cells, thus showing a similar pattern of fluorescence as free GFP (Fig. 1). These data suggest that the distribution of GFP fluorescence in punctuate structures observed upon expression of the XopJ:: GFP wild-type protein is associated with both its catalytic activity and the presence of a functional N-myristoylation motif.

Integrity of the effector::GFP fusion proteins was verified by Western blotting, using anti-GFP antibodies. As shown in Supplementary Figure S1, all three fusion proteins migrate at the expected molecular mass of approximately $70 \mathrm{kDa}$, indicating their intactness.

To test on the biochemical level whether the XopJ N-myristoylation motif confers membrane association, we conducted subcellular fractionation experiments on extracts from tissue transiently expressing XopJ::GFP, XopJ(C235A)::GFP, or XopJ (G2A)::GFP. An approximately $70-\mathrm{kDa}$ band corresponding to the predicted size of XopJ::GFP was detected using anti-GFP antiserum in total tissue extracts after low-speed centrifugation (Fig. 2A). Fractionation of these extracts with ultraspeed centrifugation showed that, in accordance with the subcellular localization data, the XopJ::GFP signal was exclusively found in the insoluble fraction corresponding to membranous particles. The same distribution pattern was found for the $\mathrm{XopJ}(\mathrm{C} 235 \mathrm{~A}):$ GFP protein (Fig 2B). In contrast, the $\mathrm{XopJ}(\mathrm{G} 2 \mathrm{~A}):$ :GFP variant, lacking a functional $\mathrm{N}$-myristoylation signal, yielded bands of nearly identical intensity in the membrane fraction and in the soluble fraction (Fig. 2C). These data indicate that N-myristoylation contributes but does not solely account for membrane association of XopJ. Interestingly, XopJ(G2A)::GFP appeared to migrate as two bands of closely similar molecular mass (Fig. 2C). This might indicate that the protein undergoes a post-translational modification different from $\mathrm{N}$-myristoylation.

To monitor proper separation of subcellular fractions during centrifugation, all blots were additionally probed with antibodies directed against sucrose-phosphatase, a soluble protein residing in the cytosol (Chen et al. 2005), and plasma membrane $\mathrm{H}^{+}$-ATPase (Morsomme et al. 1998).

\section{XopJ interferes with host-cell protein secretion.}

Increasing evidence suggests that the intracellular vesicle trafficking and polarized secretion pathways are important for basal defense reactions against bacterial pathogens (Kalde et al. 2007) and that bacterial type III effectors may be targeting the intracellular trafficking pathway to suppress cell wall-based defense responses (Nomura et al. 2006). The above findings prompted us to investigate whether XopJ would interfere with protein secretion in plant cells. To this end, we used an assay based on intracellular accumulation of a GFP variant, secGFP, that normally is secreted into the apoplast (Batoko et al. 2000). When secreted, detectable GFP fluorescence is generally very weak (Fig. 3A), but it accumulates and is visualized readily in the endomembrane system when transport to the apoplast is inhibited by a well-known inhibitor of secretion, the fungal toxin brefeldin A (Fig. 3F). As a positive control for a protein interfering with secretion, a dominant negative variant of the plasma membrane target-soluble $N$-ethylmaleimide sensitivefactor attachment protein receptors (t-SNARE) AtSYP121 lacking the C-terminal transmembrane domain was used (Tyrrell et al. 2007). Previous studies showed that expression of this cytosolic t-SNARE fragment (so-called Sp2) in plant cells selectively suppresses late steps in secretory traffic to the plasma membrane (Geelen et al. 2002; Tyrrell et al. 2007). Agrobacterium-mediated transfection by infiltration was used to transiently coexpress secGFP with AtSYP121-Sp2::myc or XopJ:: myc in leaves of $N$. benthamiana plants. secGFP accumulation was monitored by confocal laser scanning microscopy in epidermal cells $48 \mathrm{~h}$ after inoculation. Expression of the respective proteins was confirmed by gel-blot analysis of the transfected leaves (data not shown).

Expression of secGFP alone resulted in no intracellular accumulation and a weak apoplastic fluorescence that was detected only at high magnification (Fig. 3A). Coexpression of secGFP with XopJ::myc, however, led to a large increase in secGFP fluorescence that was visible clearly within the epidermal cells, forming an intracellular reticulate pattern of fluorescence (Fig 4B). A strikingly similar pattern of secGFP fluorescence was also visible in cells coexpressing secGFP with AtSYP121Sp2::myc (Fig. 3C). Thus, XopJ appears to interfere with protein secretion in a manner similar to that of AtSYP121-Sp2. In order to investigate whether the effect of XopJ on protein secretion depends on a functional catalytic triad, secGFP was cotransfected with the $\mathrm{XopJ}(\mathrm{C} 235 \mathrm{~A}):$ :myc mutant and GFP fluorescence was monitored as above. As shown in Figure 3D, expression of the XopJ variant disrupted in the catalytic triad did not affect secGFP trafficking to the apoplast.

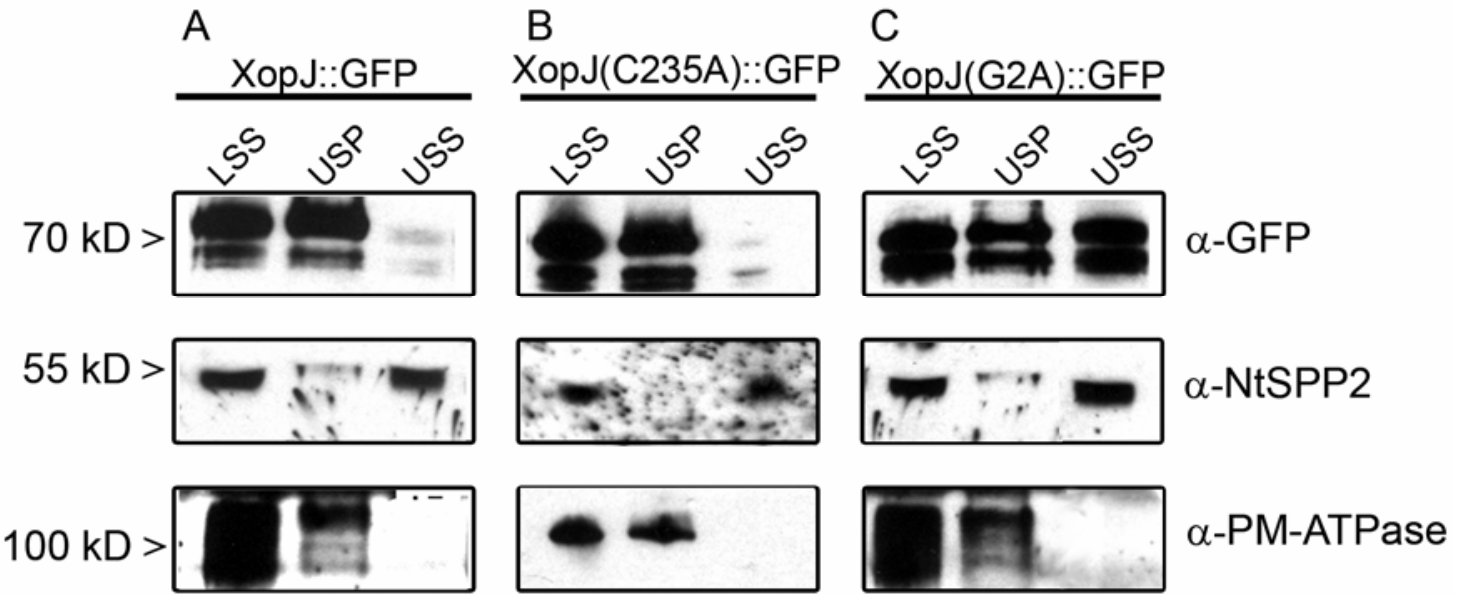

Fig. 2. Protein extracts from Nicotiana benthamiana leaves transiently expressing A, XopJ::GFP (green fluorescent protein), B, XopJ(C235A)::GFP, or C, $\mathrm{XopJ}(\mathrm{G} 2 \mathrm{~A}):$ :GFP were fractionated by ultracentrifugation. Equal volumes of the individual fractions representing approximately equal protein amounts were immunoblotted against anti-GFP antiserum. To ensure proper separation of soluble and membrane fractions, the same blots were reprobed with antibodies against sucrose-phosphatase $\left(\alpha\right.$-NtSPP2) and plasma membrane $\mathrm{H}^{+}$-ATPase ( $\alpha$-PM-ATPase). LSS $=$ low-speed supernatant containing soluble fraction and membrane fraction, USP $=$ ultraspeed pellet containing membranes, USS $=$ ultraspeed supernatant representing the soluble fraction. 
To obtain biochemical evidence that XopJ blocks protein secretion, we collected apoplastic fluid from leaves of $N$. benthamiana plants expressing secGFP and which were Agrobacterium-infiltrated to transiently express XopJ::myc or one of
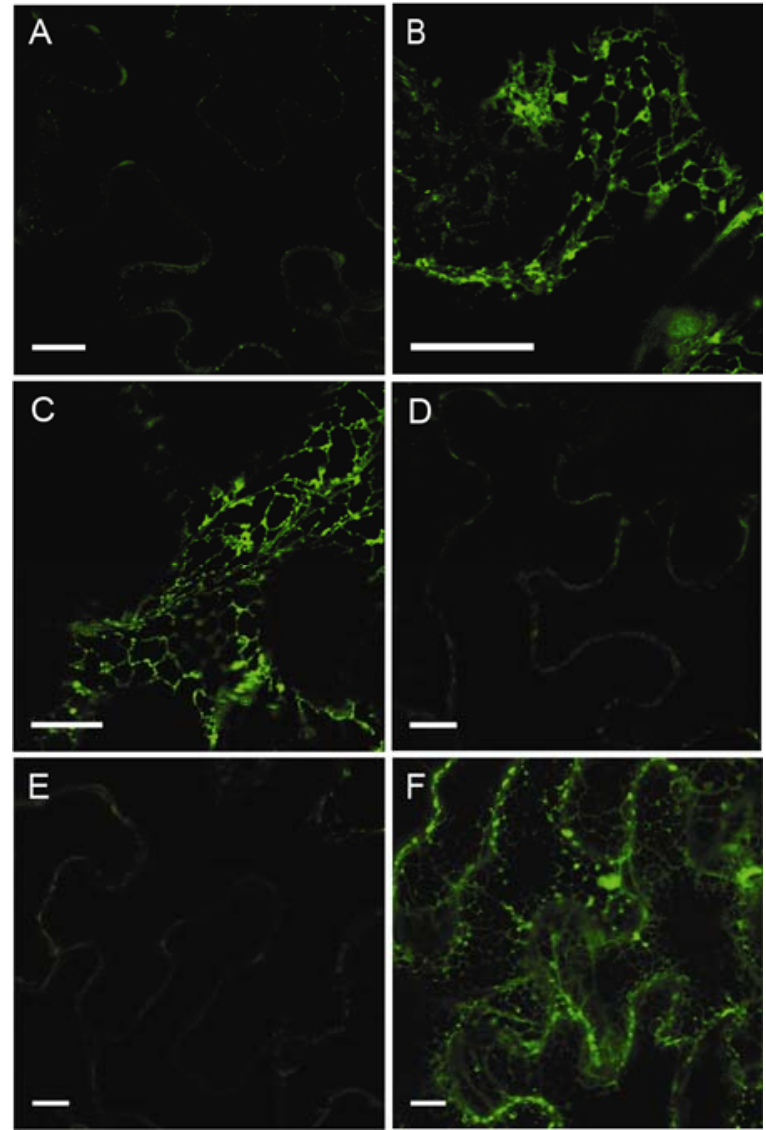

Fig. 3. Expression of XopJ blocks secretion and leads to the accumulation of secreted green fluorescent protein (secGFP) within a cytosolic reticulum. Confocal images of Nicotiana benthamiana leaf epidermis transfected to express A, secGFP alone and $\mathbf{B}$, together with XopJ::myc. $\mathbf{C}$, The effect of the cytosolic mutant of the syntaxin AtSYP121::myc on secGFP secretion. D, Coexpression of secGFP together with the XopJ (C235A)::myc mutant disrupted in the catalytic triad. E, secGFP-expressing leaf infiltrated with $0.001 \%$ dimethyl sulfoxide (DMSO). F, secGFPexpressing leaf infiltrated with brefeldin A ( $10 \mu \mathrm{g} / \mathrm{ml}$ in $0.001 \%$ DMSO). Photos in $\mathrm{E}$ and $\mathrm{F}$ were taken 20 min after infiltration. its variants. The presence of secGFP in the apoplastic fluid was monitored by immunoblotting with anti-GFP antibodies. The analysis confirmed that the level of secGFP accumulating in the apoplastic fluid was reduced when the reporter was coexpressed with XopJ::myc (Fig. 4). The fact that GFP was easily detected in the remaining tissue after isolation of extractable apoplastic proteins indicated that the protein was efficiently expressed but not secreted in the presence of XopJ::myc. Coexpression of AtSYP121::myc together with secGFP led to similar results, further supporting the notion that reduced levels of GFP in the apoplast are due to a block in secretion (Fig. 4). In contrast, appreciable amounts of secGFP could be detected in apoplastic fluid collected from leaves coexpressing the XopJ(C235A)::myc mutant, implying that a functional catalytic triad is necessary for XopJ to affect secretion (Fig. 5).

We next sought to investigate whether XopJ would specifically affect protein trafficking to the plasma membrane or whether traffic to the vacuole would also be compromised. To this end, the cationic amino-acid transporter 2 (CAT2) from Arabidopsis (Su et al. 2004) fused to tdTomato was used as a tonoplast marker. The CAT2::tdTomato fusion protein was transiently expressed in leaves of $N$. benthamiana either alone or together with XopJ::myc. Expression of CAT2::tdTomato on its own yielded a characteristic labeling ( $\mathrm{Su}$ et al. 2004), with a small cytoplasmic region including chloroplasts separating the CAT2::tdTomato-dependent fluorescence from the plasma membrane, clearly indicating tonoplast localization of the fusion protein (Fig. 5A). Coexpression with XopJ::myc yielded a very similar pattern of labeling (Fig. 5B), indicating that the plasma membrane-associated XopJ did not interfere with CAT2 traffic to the tonoplast. Thus, it can be concluded
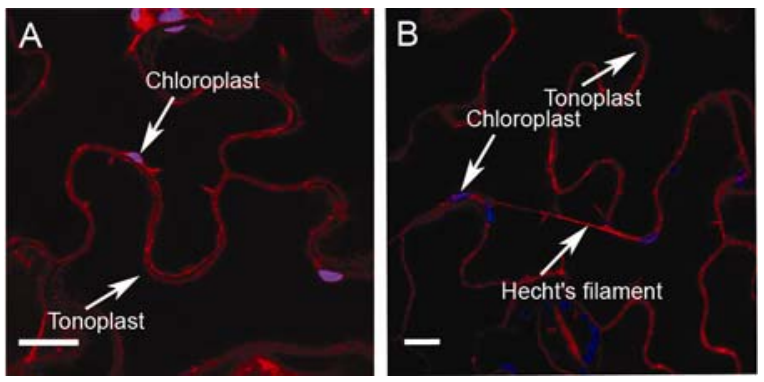

Fig. 5. XopJ does not affect traffic of CAT2::tdTomato to the tonoplast Nicotiana benthamiana leaves were infiltrated with Agrobacteria carrying either A, CAT2::tdTomato alone or B, together with XopJ.

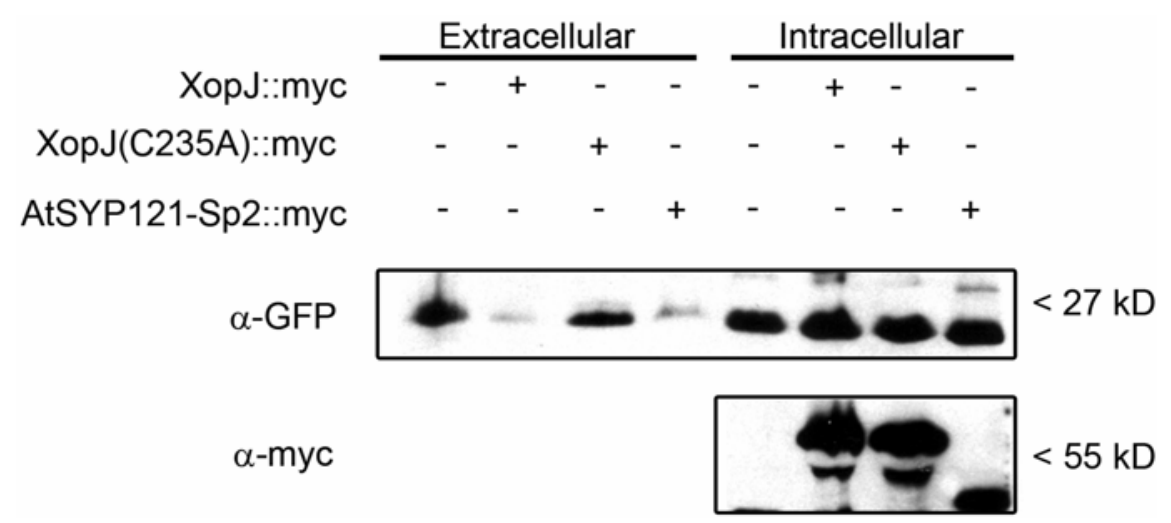

Fig. 4. Transient expression of XopJ::myc prevents accumulation of secreted green fluorescent protein (secGFP) in the apoplastic fluid of secGFP-expressing Nicotiana benthamiana plants. Leaves of $N$. benthamiana plants expressing secGFP were infiltrated with Agrobacterium strains that mediate T-DNA-based transfer of XopJ::myc, XopJ(C235A)::myc, and AtSYP121-Sp2::myc. Apoplastic fluid isolated from infiltrated leaves at 48 h postinoculation and intracellular proteins from $0.5-\mathrm{cm}^{2}$ disks of the remaining leaf tissue were separated by sodium dodecyl sulfate-polyacrylamide gel electrophoresis and were subjected to immunoblot analysis using an anti-GFP antiserum. To show expression of effector proteins, the blot was stripped and was subsequently probed with anti-myc antibody. 
that XopJ is effective in suppressing secGFP traffic to the plasma membrane but does not generally interfere with protein sorting, e.g., to the vacuole, and that the secretory block posed by XopJ is not due to an unspecific stress response resulting from overexpression of the effector.

\section{Transgenic expression of XopJ}

in Arabidopsis suppresses cell-based extracellular defense.

The ability of XopJ to block secretion of the secGFP reporter protein suggests that XopJ may be involved in the inhibition of a host-cell vesicle trafficking pathway. Accelerated vesicle traffic is associated with a polarized cell wall-associated defense in plants (Hückelhoven 2007; Robatzek 2007). One such defense response is the deposition of callose-rich papillae at the cell wall (Brown et al. 1995). Perception of PAMPs present on nonpathogens such as T3SS-deficient bacterial mutants leads to the deposition of papillae that can be visualized by aniline blue-staining of callose. In order to monitor the effect of XopJ on PAMP-triggered callose deposition, we created transgenic Arabidopsis plants expressing XopJ under control of the ethanol-inducible promoter. At $24 \mathrm{~h}$ after induction with ethanol, the plants were infected with a Pseudomonas syringae pv. tomato DC3000 hrcC mutant lacking a functional T3SS. After inoculation with the $h r c C$ mutant, Col-0 leaves exhibited characteristic callose deposits (Fig. 6) when stained with aniline blue. In contrast, XopJ-expressing plants exhibited a significant reduction in callose deposits by 50 to $90 \%$ of the number of callose deposits observed in Col-0 leaves (Fig. 6). In the transgenics, this effect was clearly dependent on the induction of XopJ expression with ethanol, while ethanol treatment had no effect on callose deposition in $h r c C$-infected Col-0 plants.

\section{DISCUSSION}

Type III effector proteins delivered into the host cell by plant-pathogenic bacteria are important virulence determinants. Accumulating evidence suggests that a major role of these effectors in promoting virulence is the suppression of host defense responses (Grant et al. 2006; Mudgett 2005). However, the specific molecular mechanisms through which individual type III effectors function to suppress defenses are still poorly understood. The study of effector function is hampered by the typically weak contributions they individually make to virulence. Deletion of a single effector gene does not often lead to a noticeable loss of virulence as measured by attenuation of bacterial growth and symptom development in infected tissue. The $X$. campestris pv. vesicatoria type III effector protein XopJ has been shown to be dispensable for bacterial virulence in a compatible interaction under laboratory conditions (Noël et al. 2003). By using transient plant-expression techniques together with a combination of cytological, biochemical, and molecular methods, we were able to provide evidence that XopJ inhibits basal defense responses by selectively affecting vesicle transport to the plasma membrane.

Examination of the subcellular localization of XopJ in plant cells revealed that a XopJ::GFP fusion protein appears to be restricted to the plasma membrane, a localization that has previously been shown to be an important site targeted by bacterial type III effectors. Several $P$. syringae type III effectors (AvrRpm1, AvrB, AvrPto, HopF2, and the entire HopZ family with the exception of HopZ3) have been found to contain $\mathrm{N}$ myristoylation sites mediating association of the proteins with the plasma membrane. This subcellular localization in plant cells appears to be important for their avirulence and virulence functions (Lewis et al. 2008; Nimchuk et al. 2000; RobertSeilaniantz et al. 2006; Shan et al. 2000). In accordance with previous observations (Thieme et al. 2007), we found that mutation of a predicted $\mathrm{N}$-myristoylation signal attenuates membrane localization of XopJ. However, fractionation experiments revealed that a considerable proportion of $\mathrm{XopJ}(\mathrm{G} 2 \mathrm{~A}):$ GFP remained within the membrane fraction. This could either be due to an additional post-translational modification of XopJ mediating membrane association, e.g., palmitoylation via thioesther bonds, or by binding of XopJ to membrane-bound target proteins. The XopJ $\mathrm{N}$ terminus contains a cysteine residue at position 4 that could become a target for palmitoylation (Thieme et al. 2007). We used mutational analysis to assess the role of this residue in plasma membrane localization of XopJ; however, a C4A mutation appeared to render the protein unstable, as we were not able to detect any expression of XopJ (C4A) in transfected N. benthamiana leaves (data not shown). On the other hand, localization of effector targets to the plasma membrane has already been demonstrated for Pto, RIN4, and PBS1, the respective plant targets of AvrPto, AvrRpt2, and
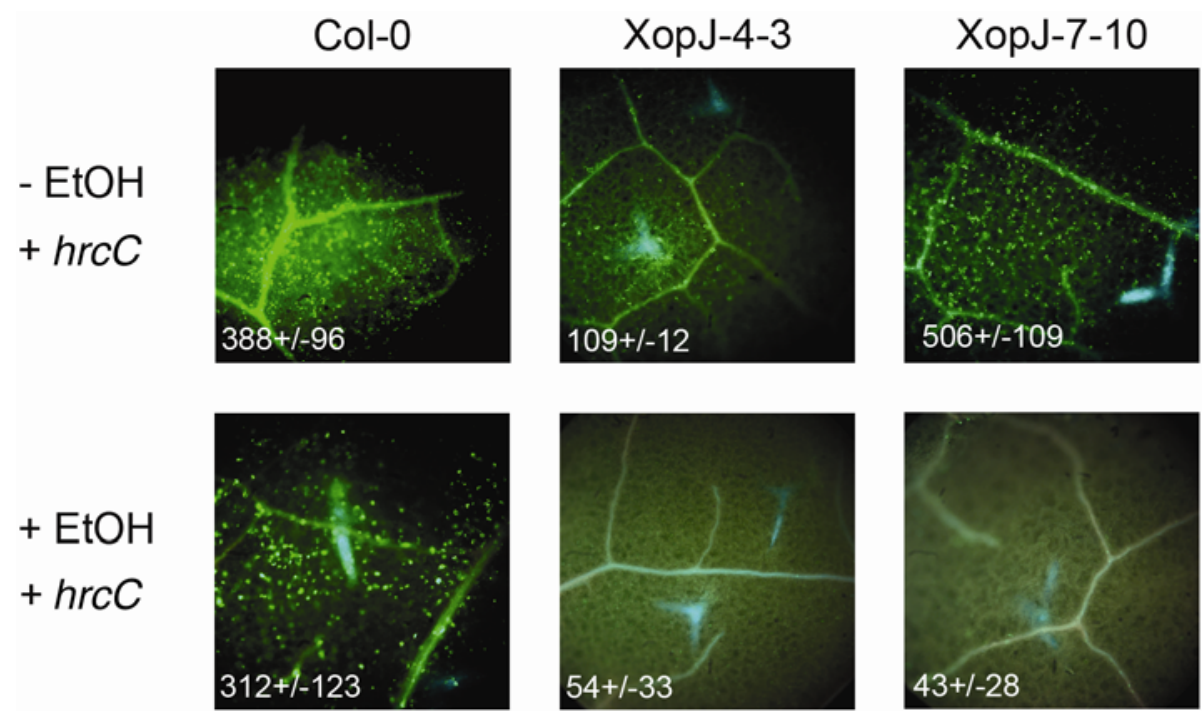

Fig. 6. Papilla-associated callose deposition in XopJ-expressing Arabidopsis plants. Col-0 and alc-XopJ plants were treated with $1 \%$ ethanol and, after 24 h, were inoculated with Pseudomonas syringae pv. tomato DC3000 hrcC mutant bacteria $\left(1 \times 10^{8} \mathrm{CFU} \mathrm{ml}^{-1}\right)$. Leaf tissue was collected after $24 \mathrm{~h}$ and was stained for callose. Plants without ethanol treatment served as control. For each treatment, the average number of callose depositions per field of view \pm standard error $(n=4)$ is shown in the lower left corner of the image. 
AvrPphB (Axtell and Staskawicz 2003; Nimchuk et al. 2000; Shao et al. 2003).

Using secGFP as an apoplastic marker, we were able to demonstrate that expression of XopJ leads to an accumulation of GFP fluorescence within the endomembrane system and to a block of secGFP secretion into the apoplast. In addition, ethanol-inducible XopJ expression in leaves of transgenic Arabidopsis strongly compromised in the ability to deposit callose associated with papillae, a hallmark of cell wall-associated defense, in response to inoculation with the nonpathogenic $P$. syringae pv. tomato DC3000 hrcC mutant. Thus, our data provide strong evidence for a role of XopJ in compromising vesicle trafficking to the plasma membrane and thereby inhibiting basal defenses.

Increasing evidence indicates that protein secretion appears to be an important step mediating plant resistance against fungal and bacterial pathogens (Hückelhoven 2007; Kalde et al. 2007; Nomura et al. 2006; Robatzek 2007; Wang et al. 2005) and that pathogen virulence factors may be targeting intracellular trafficking to suppress host immunity. Indeed, the $P$. syringae effector protein HopM1 was recently shown to localize to the plant cell's endomembrane fraction (Nomura et al. 2006). It was further demonstrated that HopM1 physically interacts with AtMIN7, one of the eight guanine nucleotide exchange factor (GEF) proteins that activate ARF GTPases in Arabidopsis, and mediates its degradation through the $26 \mathrm{~S}$ proteasome (Nomura et al. 2006). ARF-GEF proteins play a role in the recruitment of vesicle coats necessary for vesicle budding and cargo selection and, thus, are important regulators of intracellular vesicle trafficking in eukaryotic cells (Donaldson and Jackson 2000). This suggests that $P$. syringae HopM1 prevents secretion of antimicrobial peptides and compounds through modulation of early steps of vesicle trafficking by targeted ARF-GEF degradation.

Given the plasma membrane localization of XopJ in plant cells, it appears likely for XopJ to target a step in vesicle trafficking different from that affected by HopM1. The late steps of vesicle trafficking involve docking and subsequent fusion of the donor vesicle with the target membrane. This is mediated through the cooperative action of several proteins, including syntaxins, which are tSNARE. Functional classification divides SNARE into vesicle-associated (v-SNARE) and t-SNARE (Söllner et al. 1993). The t-SNARE complex serves as a binding site for $\mathrm{V}$-SNARE on the transport vesicle, and association of the v-SNARE with the t-SNARE complex is thought to drive membrane fusion (McNew et al. 2000). Intriguingly, two t-SNARE have been implicated in mediating defense-related secretion in plants, SYP132 for resistance to bacteria (Kalde et al. 2007) and SYP121 for resistance against powdery mildew fungus (Collins et al. 2003). Silencing of SYP132 in N. benthamiana plants expressing the Pto resistance gene inhibited secretion of pathogenesis-related proteins into the cell wall and compromised resistance against $P$. syringae pv. tabaci (avrPto), allowing bacteria to grow nearly to levels found in the compatible interaction (Kalde et al. 2007). We were able to show that transient expression of a dominant negative variant of the plasma membrane t-SNARE AtSYP121 (Tyrrell et al. 2007) affected secretion of the GFP reporter in a way similar to that of XopJ, as both proteins gave rise to strikingly similar secGFP fluorescence patterns and similarly affected secGFP accumulation in the apoplast. However, expression of XopJ did not alter targeting of a tonoplast marker (CAT2::tdTomato), providing additional evidence for a specific effect of XopJ on vesicle fusion at the plasma membrane. A similar site of action has been proposed for the $P$. syringae effector AvrPto, which has been identified as a suppressor of papillae-associated extracellular defense responses (Hauck et al. 2003). AvrPto is tethered to the plasma membrane by N-myristoylation and, in a search for host cell targets, AvrPto has been shown to interact with two Ras-related small $\mathrm{G}$ proteins that are involved in vesicular trafficking (Bogdanove and Martin 2000). Although a direct effect of AvrPto on protein secretion has not yet been shown, one mechanism by which this type III effector could act to suppress cell wall-based plant defense would be to inhibit an extracellular vesicle trafficking pathway by inhibiting the fusion of transport vesicles with the plasma membrane. Whether XopJ also interacts with Ras-related small G proteins as AvrPto does or with other factors of vesicle trafficking, e.g., SNARE, remains to be determined.

Taken together, the present study defines a new virulence function of XopJ that requires an intact catalytic triad. The close phylogenetic relationship between XopJ from X. campestris pv. vesicatoria and the HopZ effector family of $P$. syringae opens the possibility that these type III effectors may be functionally related homologs (Ma et al. 2006).

The identification of XopJ target proteins will not only reveal novel mechanisms by which bacterial pathogens modulate hostcell metabolism but also should provide unique insights into the regulation of protein secretion in plants.

\section{MATERIALS AND METHODS}

\section{Plasmid construction.}

XopJ expression constructs were generated using Gateway cloning technology (Invitrogen, Karlsruhe, Germany). To this end, the entire open reading frame (ORF) of XopJ (GenBank accession number CAJ23833) was amplified by polymerase chain reaction (PCR), using genomic DNA of Xanthomonas campestris pv. vesicatoria 85-10 as a template. The resulting fragment was inserted into the pENTR-D/TOPO vector according to the manufacture's instructions (Invitrogen). Subsequently, the XopJ coding region was recombined into the technique-specific destination vector, using L/R-Clonase (Invitrogen). For C-terminal GFP fusions the destination vector was pK7WGF2 (Karimi et al. 2002). To create a C-terminal fusion between XopJ and a triple myc tag, pRB35S-3xmyc was used as a destination vector that is a derivative of pPZP200 (Hajdukiewicz et al. 1994). To generate the secreted version of GFP, a fragment comprising the basic chitinase signal sequence and the coding region of GFP was amplified by PCR, using primers FB495 (5'-CACCAACAATGAAGACTAATCTTTTT CTCTTTC-3') and FB 496 (5'-TTTGTATAGTTCATCCATG CC-3') and mGFP5 (Haseloff et al. 1997) as a template. The fragment was finally inserted into pRB35S-3xmyc as described above. The AtSYP121 Sp2-expression construct in pRB35S$3 x m y c$ was generated as described (Tyrrell et al. 2007).

tdTomato was amplified by PCR from pAN459 (gift from A. Nebenführ, University of Tennessee, Knoxville, U.S.A.). Recognition sites for AvrII and PacI were introduced into the forward primer (5'-CCTAGGATGGTGAGCAAGGGCGAGGAG GTCATC-3') and reverse primer (5'-TTAATTAATTACTTGT ACAGCTCGTCCATGC-3'), respectively. The resulting product was cloned into pTopo pCR BluntII (Invitrogen) and was sequenced. The fragment was released using the AvrII and PacI and was introduced into pEARLEYGATE100 (Earley et al. 2006) digested with the same enzymes to yield the destination vector pASX. AtCAT2 was previously shown to localize to the tonoplast (Su et al. 2004). The AtCAT2 ORF was amplified from flower cDNA using primers AtCAT2c1fwd (5'-CACCAT GGGGTTTTTGGTGGATACGCAAAAGG-3') and AtCAT2c1884rev (5'-GGATCMTGCCAAAGAACCTTCATGTTCG-3'), was introduced into the vector pENTR-D/Topo (Invitrogen), and was sequenced. Subsequently, the cDNA was introduced into $\mathrm{pASX}$ by $\mathrm{L} / \mathrm{R}$ recombination. 
Site-directed mutagenesis.

The cysteine 235 to alanine substitution in XopJ was introduced using the Quick-change site-directed mutagenesis kit (Stratagene, Heidelberg, Germany), using primers XopJC235A Fwd (5'-CAGAAGTCTGCAGCGGACGCCCTGATGTTCGA TCTGCAT-3') and XopJC235A_Rev (ATGCAGATCGAACA TCAGGGCGTCCGCTGCAGACTCTG-3') according to the manufacture's instructions. The $\mathrm{XopJ}(\mathrm{G} 2 \mathrm{~A})$ mutant was generated by PCR using primers VB07 (5'-CACCGTAACAAT GGCTCTATGCGTTTCAAG-3) and VB10 (5'-GGATCCTGA CTGGCGATCAGAGATAGC- $3^{\prime}$ ). In both cases, the plasmid pENTR-XopJ was used as a template. All base changes were verified by DNA sequencing.

\section{Agroinfiltration.}

For infiltration of Nicotiana benthamiana leaves, A. tumefaciens $\mathrm{C} 58 \mathrm{C} 1$ was infiltrated into the abaxial air space of 4- to 6-week-old plants, using a needleless 2 -ml syringe. Agrobacteria were cultivated overnight at $28^{\circ} \mathrm{C}$ in the presence of appropriate antibiotics. The cultures were harvested by centrifugation, and the pellet was resuspended in sterile water to a final optical density at $600 \mathrm{~nm}\left(\mathrm{OD}_{600}\right)$ of 0.1 for the secretion assays or $\mathrm{OD}_{600}$ of 1.0 for all other experiments. The cells were used for the infiltration directly after resuspension.

\section{Colocalization and confocal laser scanning microscopy.}

Colocalization experiments were performed using the mCherry-labeled plasma membrane marker (PM-rk; CD31007), the Golgi marker (G-rk; CD3-967), and the endoplasmic reticulum marker (ER-rk; CD3-959) purchased from the Arabidopsis Biological Resource Center (Nelson et al. 2007).

Fluorescence in epidermal cells of the abaxial leaf side was assessed 2 days postinfiltration. Pieces of the infiltrated leaf were sampled randomly from the infected area and were mounted in water for observation under the microscope. In these experiments a Leica TCS SP2 (Leica, Microsystems, Bensheim, Germany) confocal laser scanning microscope was used, and images are displayed as maximum projections of picture stacks, except for closeup views of reticulate localization, which are single sections. GFP and mCherry were excited with a $20 \mathrm{~mW}$ Argon laser at 488 and $543 \mathrm{~nm}$ respectively, and the emission filter wavelengths were 497 to $526 \mathrm{~nm}$ for GFP and 570 to $615 \mathrm{~nm}$ for mCherry. tdTomato was dissected using 514-nm excitation and emission in a range of 546 to $624 \mathrm{~nm}$. Double-labeled cells were scanned sequentially to prevent any crosstalk between fluorescent channels.

\section{Membrane fractionation.}

Infected leaf material $(1 \mathrm{~g})$ was ground in $3 \mathrm{ml}$ of ice-cold extraction buffer (20 mM HEPES, $\mathrm{pH} 7.5,100 \mathrm{mM} \mathrm{NaCl}, 5$ $\mathrm{mM} \mathrm{MgCl}, 1 \mathrm{mM}$ dithiothreitol), and the homogenate was centrifuged at $2,000 \times g$ for 5 min to yield the low-speed supernatant (LSS). LSS (1 ml) was subjected to ultraspeed centrifugation at $100,000 \times g$ for $1 \mathrm{~h}$ at $4^{\circ} \mathrm{C}$. The resulting pellet (ultraspeed pellet) containing the membrane fraction was resuspended in $2 \mathrm{ml}$ of sodium dodecyl sulfate-polyacrylamide gel electrophoresis (SDS-PAGE) loading buffer. The ultraspeed supernatant and $1 \mathrm{ml}$ of the LSS were mixed with $1 \mathrm{ml}$ of $2 \times$ SDS-PAGE and, after boiling for $10 \mathrm{~min}$ at $95^{\circ} \mathrm{C}, 20 \mu \mathrm{l}$ of each sample were subjected to gel electrophoresis.

\section{Collection of apoplastic fluid.}

For the extraction of apoplastic fluid, one or two $N$. benthamiana leaves (approximately $2 \mathrm{~g}$ fresh weight) were harvested, the midrib was removed, and the leaves were weighed and washed in water. After drying, leaves were vacuum-infiltrated with $50 \mathrm{mM}$ HEPES-KOH, $\mathrm{pH} 6.8$, for 5 to $10 \mathrm{~min}$. The leaf surface was dried, and leaves were wrapped in aluminum foil and were inserted into a 50-ml Falcon tube with the tip cut down. The Falcon tube was placed in a centrifuge tube and was centrifuged at $1,400 \times g$ for $10 \mathrm{~min}$ at $4^{\circ} \mathrm{C}$. An aliquot of $30 \mu \mathrm{l}$ of the collected apoplastic fluid was used for immunoblot analysis.

\section{Generation of transgenic Arabidopsis plants.}

The XopJ reading frame was cloned into the $\mathrm{pBin} \Delta \mathrm{AlcR}$ vector, allowing for the inducible expression of the transgene after application of ethanol (Chen et al. 2003). Tho XopJ insert was sequenced and $\mathrm{pBin} \Delta \mathrm{AlcR}-\mathrm{XopJ}$ was transformed into Agrobacterium tumefaciens C58C1 (containing the pGV2260 helper plasmid). Transformation of Arabidopsis thaliana Col-0 was carried out as previously described (Clough and Bent 1998). For selection of transgenic plants, T1 seeds were sterilized and sown onto Murashige Skoog medium (Sigma, St. Louis) supplemented with Gamborg's vitamin solution $(1: 1,000)$ and $50 \mu \mathrm{g}$ of kanamycin per milliliter. Plants were given cold treatment for 2 days to synchronize germination before incubation under a 16-h-light and 8-h-dark photoperiod $\left(150 \mu \mathrm{M} \mathrm{m}^{-2} \mathrm{~s}^{-1}\right.$ light, $\left.21^{\circ} \mathrm{C}\right)$ at $50 \%$ relative humidity. Ethanol inducible XopJ expression was confirmed by reverse transcription-PCR, after transfer of the plants to soil.

\section{Callose assay.}

Six-week-old Arabidopsis Col-0 or pBin $\Delta$ AlcR-XopJ plants were root-drenched with $10 \mathrm{ml}$ of $1 \%$ ethanol per plant and, after $24 \mathrm{~h}$, were infiltrated with a suspension of the type III secretion-defective Pseudomonas syringae DC3000 hrcC mutant (Roine et al. 1997) at a concentration of $1 \times 10^{8} \mathrm{CFU} \mathrm{ml}^{-1}$ $\left(\mathrm{OD}_{600}=0.2\right)$. Plants were left under a transparent hood, and leaves were harvested after $24 \mathrm{~h}$. Harvested leaves were cleared of pigments by treatment with ethanol and were stained with aniline blue solution $(0.005 \%$ aniline blue [Sigma] in $70 \mathrm{mM} \mathrm{K}_{2} \mathrm{HPO}_{4}, \mathrm{pH}$ 9.0). Leaves were examined with a Leica DMR microscope. The number of callose deposits was determined on four microscopic views taken from four independent leaves. The callose assays reported here were performed two times with similar results.

\section{ACKNOWLEDGMENTS}

We are indebted to U. Sonnewald for his continuous and enthusiastic support. We thank U. Bonas (Martin-Luther-Universität, Halle) for her collaboration during the initial phase of the project and M. Boutry (Université catholique de Louvain) for providing the $\mathrm{PM} \mathrm{H}^{+}$-ATPase antiserum. We would also like to thank N. Sauer (Friedrich-Alexander-Universität Erlangen-Nürnberg, Germany) for providing the confocal laser scanning microscope of his department. This work was supported in part by a grant from the Deutsche Forschungsgemeinschaft in frame of the collaborative research center "reprogramming of host cells by microbial effectors" (SFB796).

\section{LITERATURE CITED}

Alfano, J. R., and Collmer, A. 2004. Type III secretion system effector proteins: Double agents in bacterial disease and plant defense. Annu. Rev. Phytopathol. 42:385-414.

Axtell, M. J., and Staskawicz, B. J. 2003. Initiation of RPS2-specified disease resistance in Arabidopsis is coupled to the AvrRpt2-directed elimination of RIN4. Cell 112:369-377.

Batoko, H., Zheng, H. Q., Hawes, C., and Moore, I. 2000. A rab1 GTPase is required for transport between the endoplasmic reticulum and Golgi apparatus and for normal Golgi movement in plants. Plant Cell 12:2201-2218.

Bestwick, C. S., Bennett, M. H., and Mansfield, J. W. 1995. Hrp mutant of Pseudomonas syringae pv. phaseolicola induces cell wall alterations but not membrane damage leading to the hypersensitive reaction in lettuce. Plant Physiol. 108:503-516.

Bogdanove, A. J., and Martin, G. B. 2000. AvrPto-dependent Pto-interact- 
ing proteins and AvrPto-interacting proteins in tomato. Proc. Natl. Acad. Sci. U.S.A. 97:8836-8840.

Bonas, U., Stall, R. E., and Staskawicz, B. 1989. Genetic and structural characterization of the avirulence gene avrBs3 from Xanthomonas campestris pv. vesicatoria. Mol. Gen. Genet. 218:127-136.

Brown, I., Mansfield, J., and Bonas, U. 1995. Hrp genes in Xanthomonas campestris pv. vesicatoria determine ability to suppress papilla deposition in pepper mesophyll cells. Mol. Plant-Microbe Interact. 8:825-836.

Büttner D, and Bonas U 2006. Who comes first? How plant pathogenic bacteria orchestrate type III secretion. Curr. Opin. Microbiol. 9:193-200.

Chen, S., Hofius, D., Sonnewald, U., and Börnke, F. 2003. Temporal and spatial control of gene silencing in transgenic plants by inducible expression of double-stranded RNA. Plant J. 36:731-740.

Chen, S., Hajirezaei, M., Peisker, M., Tschiersch, H., Sonnewald, U., and Börnke, F. 2005. Decreased sucrose-6-phosphate phosphatase level in transgenic tobacco inhibits photosynthesis, alters carbohydrate partitioning, and reduces growth. Planta 221:479-492.

Clough, S. J., and Bent, A. F. 1998. Floral dip: A simplified method for Agrobacterium-mediated transformation of Arabidopsis thaliana. Plant J. 16:735-743.

Collins, N. C., Thordal-Christensen, H., Lipka, V., Bau, S., Kombrink, E., Qiu, J. L., Hückelhoven, R., Stein, M., Freialdenhoven, A., Somerville, S. C., and Schulze-Lefert, P. 2003. SNARE-protein-mediated disease resistance at the plant cell wall. Nature 425:973-977.

de Torres, M., Mansfield, J. W., Grabov, N., Brown, I. R., Ammouneh, H., Tsiamis, G., Forsyth, A., Robatzek, S., Grant, M., and Boch, J. 2006. Pseudomonas syringae effector AvrPtoB suppresses basal defence in Arabidopsis. Plant J. 47:368-382.

DebRoy, S., Thilmony, R., Kwack, Y. B., Nomura, K., and He, S. Y. 2004 A family of conserved bacterial effectors inhibits salicylic acid-mediated basal immunity and promotes disease necrosis in plants. Proc. Natl. Acad. Sci. U.S.A. 101:9927-9932.

Donaldson, J. G., and Jackson, C. L. 2000. Regulators and effectors of the ARF GTPases. Curr. Opin. Cell Biol. 12:475-482.

Earley, K. W., Haag, J. R., Pontes, O., Opper, K., Juehne, T., Song, K. M., and Pikaard, C. S. 2006. Gateway-compatible vectors for plant functional genomics and proteomics. Plant J. 45:616-629.

Espinosa, A., Guo, M., Tam, V. C., Fu, Z. Q, and Alfano, J. R. 2003. The Pseudomonas syringae type III-secreted protein HopPtoD2 possesses protein tyrosine phosphatase activity and suppresses programmed cell death in plants. Mol. Microbiol. 49:377-387.

Fu, Z. Q., Guo, M., Jeong, B.-r., Tian, F., Elthon, T. E., Cerny, R. L., Staiger, D., and Alfano, J. R. 2007. A type III effector ADP-ribosylates RNA-binding proteins and quells plant immunity. Nature 447:284-288.

Galán, J. E., and Wolf-Watz, H. 2006. Protein delivery into eukaryotic cells by type III secretion machines. Nature 444:567-573.

Geelen, D., Leyman, B., Batoko, H., Di Sansebastiano, G. P., Moore, I., and Blatt, M. R. 2002. The abscisic acid-related SNARE homolog NtSyr1 contributes to secretion and growth: Evidence from competition with its cytosolic domain. Plant Cell 14:387-406.

Grant, S. R., Fisher, E. J., Chang, J. H., Mole, B. M., and Dangl, J. L. 2006. Subterfuge and manipulation: Type III effector proteins of phytopathogenic bacteria. Annu. Rev. Microbiol. 60:425-449.

Gürlebeck, D., Thieme, F., and Bonas, U. 2006. Type III effector proteins from the plant pathogen Xanthomonas and their role in the interaction with the host plant. J. Plant Physiol. 163:233-255.

Hajdukiewicz, P., Svab, Z., and Maliga, P. 1994. The small, versatile pPZP family of Agrobacterium binary vectors for plant transformation. Plant Mol. Biol. 25:989-994.

Haseloff, J., Siemering, K. R., Prasher, D. C., and Hodge, S. 1997. Removal of a cryptic intron and subcellular localization of green fluorescent protein are required to mark transgenic Arabidopsis plants brightly. Proc. Natl. Acad. Sci. U.S.A. 94:2122-2127.

Hauck, P., Thilmony, R., and He, S. Y. 2003. A Pseudomonas syringae type III effector suppresses cell wall-based extracellular defense in susceptible Arabidopsis plants. Proc. Natl. Acad. Sci. U.S.A. 100:85778582.

Hotson, A., and Mudgett, M. B. 2004. Cysteine proteases in phytopathogenic bacteria: Identification of plant targets and activation of innate immunity. Curr. Opin. Plant Biol. 7:384-390.

Hückelhoven, R. 2007. Transport and secretion in plant-microbe interactions. Curr Opin Plant Biol 10:573-579.

Janjusevic, R., Abramovitch, R. B., Martin, G. B., and Stebbins, C. E. 2006. A bacterial inhibitor of host programmed cell death defenses is an E3 ubiquitin ligase. Science 311:222-226.

Kalde, M., Nühse, T. S., Findlay, K., and Peck, S. C. 2007. The syntaxin SYP132 contributes to plant resistance against bacteria and secretion of pathogenesis-related protein 1. Proc. Natl. Acad. Sci. U.S.A. 104:1185011855.

Karimi, M., Inzé, D., and Depicker, A. 2002. GATEWAY ${ }^{\mathrm{TM}}$ vectors for
Agrobacterium-mediated plant transformation. Trends Plant Sci. 7:193195.

Kay, S., Hahn, S., Marois, E., Hause, G., and Bonas, U. 2007. A bacterial effector acts as a plant transcription factor and induces a cell size regulator. Science 318:648-651.

Lewis, J. D., Abada, W., Ma, W., Guttman, D. S., and Desveaux, D. 2008. The HopZ family of Pseudomonas syringae type III effectors require myristoylation for virulence and avirulence functions in Arabidopsis thaliana. J. Bacteriol. 190:2880-2891.

Ma, W., Dong, F. F., Stavrinides, J., and Guttman, D. S. 2006. Type III effector diversification via both pathoadaptation and horizontal transfer in response to a coevolutionary arms race. PLoS Genet 2:e209. Published online.

Marois, E., Van den Ackerveken, G., and Bonas, U. 2002. The Xanthomonas type III effector protein AvrBs3 modulates plant gene expression and induces cell hypertrophy in the susceptible host. Mol. Plant-Microbe Interact. 15:637-646.

McNew, J. A., Parlati, F., Fukuda, R., Johnston, R. J., Paz, K., Paumet, F. Söllner, T. H., and Rothman, J. E. 2000. Compartmental specificity of cellular membrane fusion encoded in SNARE proteins. Nature 407:153-159.

Morsomme, P., Dambly, S., Maudoux, O., and Boutry, M. 1998. Single point mutations distributed in 10 soluble and membrane regions of the Nicotiana plumbaginifolia plasma membrane PMA2 $\mathrm{H}^{+}$-ATPase activate the enzyme and modify the structure of the $\mathrm{C}$-terminal region. $\mathrm{J}$. Biol. Chem. 273:34837-34842.

Mudgett, M. B. 2005. New insights to the function of phytopathogenic bacterial type III effectors in plants. Annu Rev Plant Biol 56:509-531.

Mukherjee, S., Hao, Y. H., and Orth, K. 2007. A newly discovered posttranslational modification-The acetylation of serine and threonine residues. Trends Biochem. Sci. 32:210-216.

Mukherjee, S., Keitany, G., Li, Y., Wang, Y., Ball, H. L., Goldsmith, E. J., and Orth, K. 2006. Yersinia YopJ acetylates and inhibits kinase activation by blocking phosphorylation. Science 312:1211-1214.

Nelson, B. K., Cai, X., and Nebenführ, A. 2007. A multicolored set of in vivo organelle markers for co-localization studies in Arabidopsis and other plants. Plant J. 51:1126-1136.

Nimchuk, Z., Marois, E., Kjemtrup, S., Leister, R. T., Katagiri, F., and Dangl, J. L. 2000. Eukaryotic fatty acylation drives plasma membrane targeting and enhances function of several type III effector proteins from Pseudomonas syringae. Cell 101:353-363.

Noël, L., Thieme, F., Gabler, J., Büttner, D., and Bonas, U. 2003. XopC and XopJ, two novel type III effector proteins from Xanthomonas campestris pv. vesicatoria. J. Bacteriol. 185:7092-7102.

Nomura, K., Debroy, S., Lee, Y. H., Pumplin, N., Jones, J., and He, S. Y. 2006. A bacterial virulence protein suppresses host innate immunity to cause plant disease. Science 313:220-223.

Oh, H. S., and Collmer, A. 2005. Basal resistance against bacteria in Nicotiana benthamiana leaves is accompanied by reduced vascular staining and suppressed by multiple Pseudomonas syringae type III secretion system effector proteins. Plant J. 44:348-359.

Orth, K. 2002. Function of the Yersinia effector YopJ. Curr. Opin. Microbiol. 5:38-43.

Orth, K., Xu, Z., Mudgett, M. B., Bao, Z. Q., Palmer, L. E., Bliska, J. B., Mangel, W. F., Staskawicz, B., and Dixon, J. E. 2000. Disruption of signaling by Yersinia effector YopJ, a ubiquitin-like protein protease. Science 290:1594-1597.

Robatzek, S. 2007. Vesicle trafficking in plant immune responses. Cell Microbiol. 9:1-8.

Robert-Seilaniantz, A., Shan, L., Zhou, J.-M., and Tang, X. 2006. The Pseudomonas syringae pv. tomato DC3000 type III effector HopF2 has a putative myristoylation site required for its avirulence and virulence functions. Mol. Plant-Microbe Interact. 19:130-138.

Roden, J., Eardley, L., Hotson, A., Cao, Y., and Mudgett, M. B. 2004. Characterization of the Xanthomonas AvrXv4 effector, a SUMO protease translocated into plant cells. Mol. Plant-Microbe Interact. 17:633-643.

Roine, E., Wei, W., Yuan, J., Nurmiaho-Lassila, E. L., Kalkkinen, N., Romantschuk, M., and He, S. Y. 1997. Hrp pilus: An hrp-dependent bacterial surface appendage produced by Pseudomonas syringae pv. tomato DC3000. Proc. Natl. Acad. Sci. U.S.A. 94:3459-3464.

Römer, P., Hahn, S., Jordan, T., Strauss, T., Bonas, U., and Lahaye, T. 2007. Plant pathogen recognition mediated by promoter activation of the pepper Bs3 resistance gene. Science 318:645-648.

Shan, L., Thara, V. K., Martin, G. B., Zhou, J. M., and Tang, X. 2000. The pseudomonas AvrPto protein is differentially recognized by tomato and tobacco and is localized to the plant plasma membrane. Plant Cell 12:2323-2338

Shao, F., Golstein, C., Ade, J., Stoutemyer, M., Dixon, J. E., and Innes, R. W. 2003. Cleavage of Arabidopsis PBS1 by a bacterial type III effector. Science 301:1230-1233. 
Söllner, T., Whiteheart, S. W., Brunner, M. , Erdjument-Bromage, H., Geromanos, S., Tempst, P., and Rothman, J. E. 1993. SNAP receptors implicated in vesicle targeting and fusion. Nature 362:318-324.

Staskawicz, B. J. 2001. Genetics of plant-pathogen interactions specifying plant disease resistance. Plant Physiol. 125:73-76.

Su, Y.-H., Frommer, W. B., and Ludewig, U. 2004. Molecular and functional characterization of a family of amino acid transporters from Arabidopsis. Plant Physiol. 136:3104-3113.

Thieme, F., Koebnik, R., Bekel, T., Berger, C., Boch, J., Büttner, D., Caldana, C., Gaigalat, L., Goesmann, A., Kay, S., Kirchner, O., Lanz, C., Linke, B., McHardy, A. C., Meyer, F., Mittenhuber, G., Nies, D. H., NiesbachKlosgen, U., Patschkowski, T., Rückert, C., Rupp, O., Schneiker, S., Schuster, S. C., Vorholter, F. J., Weber, E., Pühler, A., Bonas, U., Bartels, D., and Kaiser, O. 2005. Insights into genome plasticity and pathogenicity of the plant pathogenic bacterium Xanthomonas campestris pv. vesicatoria revealed by the complete genome sequence. J. Bacteriol. 187:72547266.
Thieme, F., Szczesny, R., Urban, A., Kirchner, O., Hause, G., and Bonas, U. 2007. New type III effectors from Xanthomonas campestris pv. vesicatoria trigger plant reactions dependent on a conserved N-myristoylation motif. Mol. Plant-Microbe Interact. 20:1250-1261.

Tyrrell, M., Campanoni, P., Sutter, J. U., Pratelli, R., Paneque, M., Sokolovski, S., and Blatt, M. R. 2007. Selective targeting of plasma membrane and tonoplast traffic by inhibitory dominant-negative. SNARE fragments. Plant J. 51:1099-1115.

Wang, D., Weaver, N. D., Kesarwani, M., and Dong, X. 2005. Induction of protein secretory pathway is required for systemic acquired resistance. Science 308:1036-1040.

White, F. F., Yang, B., and Johnson, L. B. 2000. Prospects for understanding avirulence gene function. Curr. Opin. Plant Biol. 3:291-298.

Xiang, T., Zong, N., Zou, Y., Wu, Y., Zhang, J., Xing, W., Li, Y., Tang, X., Zhu, L., Chai, J., and Zhou, J. M. 2008. Pseudomonas syringae effector AvrPto blocks innate immunity by targeting receptor kinases. Curr. Biol. 18:74-80. 